\title{
Ghrelin imaging probe specific for prostate cancer
}

A Canadian research team has developed a novel imaging probe for detecting prostate cancer and prostatic intraepithelial neoplasia (PIN) by synthesizing a fluorescent analogue of ghrelin - an appetite-regulating factor that binds growth hormone secretagogue receptor (GHSR) - with enhanced serum stability.

GHSR, which is responsible for the regulation of growth hormone secretion in the brain and pituitary gland, is differentially expressed in various tumours, including prostate cancer. In the study by Lu and colleagues, binding and uptake of the fluorescein-ghrelin probe were assessed ex vivo in prostate cancer cells and tissue samples from 13 patients with Gleason score 7 tumours.

Probe signal intensity was 4.7 -fold and 1.9-fold higher in regions of cancer and PIN, respectively, than in normal and BPH tissue, indicating high specificity of the probe for both malignant conditions. Although mean signal intensity for prostate cancer was 2.5 times that of PIN, this difference was not statistically significant. The study authors attributed this finding to the wide range of probe uptake values for tumours from different patients (1.5-fold to 28-fold increase over normal glands), suggesting that a ghrelinbased probe could detect disease more effectively in some patients than in others.

\section{4 ...we are optimistic that a ghrelin-based probe could be a useful diagnostic for prostate cancer... 77}

"Despite this wide range, all prostate cancers showed uptake that was greater than the highest values for normal and hyperplastic glands," says John Lewis, who led the research. "This makes the data quite compelling and we are optimistic that a ghrelin-based probe could be a useful diagnostic for prostate cancer."
Imaging tools that can reliably predict both the anatomical and biophysical characteristics of prostate cancer are urgently needed. Radiopharmaceutical probes such as ${ }^{18} \mathrm{~F}$-FDG are already in clinical use for patients with breast, colorectal and lung carcinomas, but these are of limited value for prostate cancer.

Elevated uptake of these probes in normal and $\mathrm{BPH}$ tissue frequently leads to false-negative results and areas of inflammation-which are common in the aging prostate-increase the risk of falsepositive results. "Our work suggests that fluorescein-ghrelin will not be subject to these limitations," Lewis assures. The team is currently developing a ghrelin analogue probe suitable for in vivo imaging.

Melanie Clyne

Original article Lu, C. et al. Ghrelin receptor as a novel imaging target for prostatic neoplasms. Prostate doi:10.1002/pros. 21484 